Dig Surg 1996;13:505-506

\title{
Subject Index Vol. 13, 1996
}

Abscess 231 Absorbable sutures 468 Absorption 181 Acidosis 255 Acute abdomen 487 acalculous cholecystitis 328

appendicitis 304, 456

calculous cholecystitis 328

cholecystitis 328, 332

necrotizing pancreatitis 357

pancreatitis 219, 269, 362, 365,370,374

respiratory distress syndrome 273

- - -, clinical picture

273 Acute-phase reactants 456 Adenocarcinoma 12 Adenomatosis coli 473 Adrenergic agents 245

Algorithm 374

Anastomotic complications 205 Antibiotic prophylaxis 370

- $\quad$ treatment 365

Antibiotics 287, 317, 346, 357,

374 Antimicrobial agents 421

prophylaxis 301

therapy 421 Aortic stenosis 213 APACHE II 381

Apical type, intussusception

235 Appendectomy 301,309,444 Appendicitis 287,301,309

Bacteremia 317

Bacteria 357

Bacterial pathogenesis 390

resistance 421

translocation 250, 261,269, 362

Bactibilia 317 Bariatric surgery 45 Basel type, intussusception 235 Bile 317

Biliary and pancreatic surgery 468

atresia 490

obstruction 321

- $\quad$ tract surgery 287

Biological changes 468

Body weight 19

C-reactive protein 412 Carcinoids 161 Cardiovascular changes 2 Care-related stress factors 439

Catheter-related infection 412 Cholangitis 314,317,321 Cholecystectomy 321 Cholecystitis

314,317 Cholecystokinin 6 Cholecystostomy 328 Choledocholithiasis 321 Chronic abdominal pain 231

inflammation 67

pancreatitis 79, 109, 113, 134,141, 150,209,464,495

Classification 381 Clean surgery 287 Colo-anal anastomosis 452 Colon surgery 287 Colonic diverticulitis 349

reservoir 452

Complication(s) 79, 473

-, ileostomy 26 
-, left resection 150 Computed tomography 338 Conservative approaches 309

- $\quad$ treatment 73

Contrast enema 338

Controlled randomized trial 141

Corticosteroids 205

Cost analysis 301

- $\quad$ and risk analysis 444

Critical illness 277

Crohn's disease 487

Cystic duct stump leakage 228 Cytokines 245

De novo carcinoma 59 Diabetes risk 118 Diagnosis 309,314,346 Differential diagnosis 34 Diffuse peritonitis 396 Diverticulitis 338, 346, 353 Diverticulosis 231, 338 Duct obstruction 127 Ductal disruption 90

- $\quad$ obstruction 90

Duodenal diverticulum, perfo

ration 222

Duodenum-preserving pancreatic head resection 127,141,495

- $\quad$ resection 209

Early diagnosis, duodenal diverticulum perforation 222

- $\quad$ surgery, perforated duodenal

diverticulum 222

Endogenous infections 294 Endoscopic papillotomy 228

- $\quad$ retrograde cholangio-

pancreatography 321

treatment 90 Endotoxemia 261 Endotoxin 261,384,477 Enteral feeding 410

nutrition 245 Enteric function 181 Enterocytes 250 Esophageal carcinoma 29 Esophagus 273

Etappenlavage 396 Etiology 381 Extracorporeal shock wave

lithotripsy 219

Fatal complications 118

Fever 417

-, postoperative 412

Fibrosis 134

Flowmotion 250

Focal nodular hyperplasia 483

Food intake 19

Gastric carcinoma 194

stapling 45

surgery, rat pancreas 6

ulceration 50 Gastrin 6

Gastroduodenal surgery 287 Gastrografin 201 Gastrointestinal bleeding 213

- $\quad$ intussusception 235

Gastropericardial fistula 50

Glutamine 277

Gracilis muscle 38 Gut permeability 245

Hemorrhage 170 Hepatic function 477 Hernia, anamnestic data 34 Herniography 34 Hypertrophic osteoarthropathy

490 Hypoxia 245, 255

Idiopathic gastrointestinal

bleeding 213 IgG 430

Ileoanal anastomosis 473 Ileostomy 26 Ileum 231 Imaging 338 
Immunoglobulin therapy 430 Incarcerated hernia 34 Indications 127 Infarction 483 Infected bile 362

pancreatic necrosis 370 Infection 357 Inflammatory bowel disease 26

enlargement 209 Intensive care 294,410,421,

439

- unit 417,435 Interleukin-6 412 Intestinal endometriosis 487

ischemia 170

obstruction 231

radiography 201

surgery 201 Intramucosal pH 255 Intravenous immune globulin

435 In vivo imaging 161 Ischemia 53, 67 -, pancreatic 459 Islet cell tumors 161 Isolated perfused liver 477

Jaundice 79 Jejunum 231

Kasai portoenterostomy 490 Klebsiella spp. 435

Laparoscopic cholecystectomy 228, 328, 332

Laparoscopy 2, 321, 444

Laparotomy 309

Laser-Doppler flowmetry 459

Lavage 374,400

Left resection 150

Length of hospitalization 405

Leukocyte adhesiveness/aggregation test 456

Limitations 73

Lipid peroxidation 170

Liver 483

505

Local resection 134

Low anterior resection 452

Lysosomal enzyme 477

Magnetic resonance imaging

483 Malignant fistulae 38 Mannheimer Peritonitis Index

381 Mediators 245 Medical ethics 439

- $\quad$ treatment failure 349

Meta-analyses 309

Microbiology 282

Migrating motor complex 181

Morbid obesity 45

Morbidity 332

Mortality 332, 374 Motility 181 Mucosa 250 Multiple trauma 405 Multiple-organ dysfunction syndrome 261

- $\quad$ failure 250, 277, 384, 425

Necrosectomy 374 Necrosis 357

Necrotizing pancreatitis 374 Nitric oxide 425 Nosocomial infection(s) 412, 417,435

pneumonia 405

Octreotide 161,459,464

Open cholecystectomy 328, 332

Operation, delayed 328

-, early 328

Organ preservation 495

Oxygen radicals 170 
Pain 67, 79, 109

control 464

relief 73

Palliative resection 29 Pancreas divisum 219 Pancreatectomy 12 Pancreatic cancer 12

head 134,209

pseudocyst 101,464

resection 113

sphincter dysfunction 90

surgery 101

tissue concentrations 365 Pancreat(ic)oduodenectomy

113,118

Pancreaticojejunostomy 109

Pancreatitis 101

-, experimental acute 459

Pathology 2

Pedunculated muscle bundle 59

Pelvic abscess computed tomography 349

Perforation 231

Perineal fistulae 38

Perineurium 67

Peritonitis 282, 381, 384, 390, 400

Persistent fibrosis 118

Pharmacoeconomics 405

Pharmacokinetics 365

Physical examination 34

Physiology 2

Pigs 19

Pneumonia 412

Polymorphonuclear leukocytes 170

Pouch reconstruction 194

Premedication 287

Preoperative diagnosis 127

Pressure 67

Primary anastomosis 353

Proctitis 53

Prophylaxis 410

Protease inhibitor 477

Pseudocysts 79, 90

Pseudomonas 317

- $\quad$ aeruginosa 435

Pudendal artery 53

Pulmonary changes 2

Pylorus preservation 118

Pylorus-preserving Whipple

resection 141

Radiation 38 Radiography 338 Radiotherapy 29 Ranitidine prophylaxis 405 Rectal cancer 452

- $\quad$ neck 53

Rectourethral fistulae 38 Reoperation, ileostomy 26 Reperfusion 170 Resistant pathogens 282

Reticuloendothelial system

dysfunction 362 Risk factors 405 Roux-en-Y gastric bypass 45 
Scavengers 170 Scheduled reoperations 396 Score 309

Selective decontamination 294 Sepsis 245,250,255,261,269, 314,384,425,430

score 430

syndrome 425 Septic shock 255,425,430 Serum albumin 19

protein 19 Shock 170,250 Sigmoid colon 59 Single-stage procedure 353 Small bowel obstruction 201,

235

- transplantation 181 Somatostatin receptors 161 Splanchnic blood flow 245

ischaemia 255

Standard therapy 328 Steatorrhea 73 Stress ulcer 410 Surgery 109,261,346,374 Surgical infections 430

intervention 73

stapling 205

strategy 353

technique 127

treatment 495

wound infection 287 Systemic inflammatory

response syndrome 250, 417

Taurocholate pancreatitis 459 Therapeutic concepts 396 Tissue perfusion, pancreatic 459

- $\quad$ reactions 468

Tonometry 255

Total gastrectomy 19,194 Transhiatal esophagectomy 50 Translocation 245,261 Trauma 250

Treatment 346, 390, 400

Ulcerative colitis 473 Ultrasonography 304 Ultrasound 309, 338 Upper gastrointestinal bleeding 405

Valvular heart disease 213 Vasomotion 250 Ventilator-associated pneumonia 417

Weight loss 19 Whipple operation 113

- $\quad$ resection 495

Xanthine oxidase 170

506

Subject Index Vol. 13,1996 